\title{
POISSON BOUNDARIES OF DISCRETE QUANTUM GROUPS
}

\author{
REIJI TOMATSU \\ Department of Mathematics \\ Faculty of Science Technology, Tokyo University of Science \\ Yamazaki 2641, Noda, Chiba, Japan \\ E-mail: tomatsu_reiji@ma.noda.tus.ac.jp
}

\begin{abstract}
This is a survey article about a theory of a Poisson boundary associated with a discrete quantum group. The main problem of the theory, that is, the identification problem is explained and solved for some examples.
\end{abstract}

1. Introduction. In 7], M. Izumi initiates the theory of a Poisson boundary for a random walk on a discrete quantum group. The main problem is the identification, that is, to find a more concrete realization as a von Neumann algebra. For example, the $S U_{q}(n)$ case is studied in [7, 8, and their main result shows the identification of the Poisson boundary with the quantum flag manifold $\mathbb{T} \backslash S U_{q}(n)$. This result is generalized to a co-amenable compact quantum group with commutative fusion rules by the author in [18. Starting from basics of compact or discrete quantum groups, we explain the idea of its proof.

In this article, another new problem is also presented in $\$ 4.2$. More precisely, our conjecture is the following:

CONJECTURE 1 . Let $\mathbb{G}$ be a compact quantum group and $\mu$ a generating probability measure. Then the following equality holds:

$$
H^{\infty}\left(\widehat{\mathbb{G}}, P_{\mu}\right)_{\text {class }}=Z\left(H^{\infty}\left(\widehat{\mathbb{G}}, P_{\mu}\right)\right) .
$$

Here $H^{\infty}\left(\widehat{\mathbb{G}}, P_{\mu}\right)$ class means the classical Poisson boundary on the center of the discrete quantum group, and $Z\left(H^{\infty}\left(\widehat{\mathbb{G}}, P_{\mu}\right)\right)$ is the center of the Poisson boundary. In the final

2000 Mathematics Subject Classification: Primary 46L65; Secondary 46L55.

Key words and phrases: quantum group, actions, Poisson boundary.

The paper is in final form and no version of it will be published elsewhere. 
part of this article, we will verify this conjecture for $S U_{q}(2)$. We do not know if that holds for other $q$-deformations of classical compact Lie groups, but it does for some non-amenable examples such as $A_{o}(F)$ and $A_{u}(F)$ [19, 20].

2. Quantum groups. Throughout this survey, we will mainly treat quantum groups of compact or discrete type. Our standard references are [5, 11, 22. We denote by $\otimes$ minimal tensor products or spatial tensor products for $C^{*}$-algebras or von Neumann algebras, respectively.

2.1. Compact quantum groups. The following definition of a compact quantum group has been introduced by S. L. Woronowicz [22]:

Definition 2.1 (Woronowicz). A compact quantum group (c.q.g.) $\mathbb{G}$ is a pair $(C(\mathbb{G}), \delta)$ that satisfies the following conditions:

1. $C(\mathbb{G})$ is a separable unital $C^{*}$-algebra;

2. (Coproduct) The map $\delta: C(\mathbb{G}) \rightarrow C(\mathbb{G}) \otimes C(\mathbb{G})$ is a coproduct, i.e. it is a faithful unital $*$-homomorphism satisfying the co-associativity condition,

$$
(\delta \otimes \mathrm{id}) \circ \delta=(\mathrm{id} \otimes \delta) \circ \delta ;
$$

3. (Cancellation property) The vector spaces $\delta(C(\mathbb{G}))(\mathbb{C} \otimes C(\mathbb{G}))$ and $\delta(C(\mathbb{G}))(C(\mathbb{G}) \otimes \mathbb{C})$ are dense in $C(\mathbb{G}) \otimes C(\mathbb{G})$.

EXAMPLE 2.2. A compact group $\mathbb{G}$ is regarded as a compact quantum group. Indeed, via the identification $C(\mathbb{G}) \otimes C(\mathbb{G})=C(\mathbb{G} \times \mathbb{G})$, a coproduct $\delta$ is defined by

$$
\delta(x)(r, s):=x(r s) \text { for all } x \in C(\mathbb{G}), r, s \in \mathbb{G} .
$$

The cancellation property means $r s=r t$ or $s r=t r$ imply $s=t$ for $r, s, t \in \mathbb{G}$. Note that a compact semigroup with cancellation property is a compact group.

Let $\Gamma$ be a discrete group. Then the full group $C^{*}$-algebra $\mathrm{C}^{*} \Gamma$ and the reduced group $C^{*}$-algebra $\mathrm{C}_{\text {red }}^{*} \Gamma$ are compact quantum groups. The coproducts are given by $\delta(r)=r \otimes r$ for $r \in \Gamma$.

The following state called Haar state plays an important role in the study of quantum groups.

Theorem 2.3 (Woronowicz). There exists a unique state $h \in C(\mathbb{G})^{*}$ such that

$$
(\mathrm{id} \otimes h)(\delta(a))=h(a) 1=(h \otimes \mathrm{id})(\delta(a)) \text { for all } a \in C(\mathbb{G}) .
$$

ExAmple 2.4. The Haar state of $\mathrm{C}_{\text {red }}^{*} \Gamma$ is given by the canonical tracial state $\left(\cdot \delta_{e}, \delta_{e}\right)$. Composing the state and the surjection $\mathrm{C}^{*} \Gamma \rightarrow \mathrm{C}_{\text {red }}^{*} \Gamma$, we obtain the Haar state on $\mathrm{C}^{*} \Gamma$, which is not faithful when $\Gamma$ is non-amenable.

2.2. Reduced quantum groups. Let $N_{h}:=\left\{a \in C(\mathbb{G}) \mid h\left(a^{*} a\right)=0\right\}$. Then it is known that $N_{h}$ is in fact an ideal of $C(\mathbb{G})$, and we can consider the reduced compact quantum group $C\left(\mathbb{G}_{\text {red }}\right):=C(\mathbb{G}) / N_{h}$ with a natural coproduct. By definition, $h$ is faithful on $C\left(\mathbb{G}_{\text {red }}\right)$. 
Let $\left(L^{2}(\mathbb{G}), \pi_{h}, \Omega_{h}\right)$ be the GNS representation associated with the Haar state $h$, that is,

- $L^{2}(\mathbb{G})$ is a Hilbert space;

- $\pi_{h}: C(\mathbb{G}) \rightarrow B\left(L^{2}(\mathbb{G})\right)$ is a $*$-homomorphism;

- $\Omega_{h} \in L^{2}(\mathbb{G})$ is the GNS cyclic vector, i.e. we have $L^{2}(\mathbb{G})=\overline{\pi_{h}(C(\mathbb{G})) \Omega_{h}}$ and $h(a)=\left(\pi_{h}(a) \Omega_{h}, \Omega_{h}\right)$.

Note that $N_{h}$ is precisely equal to ker $\pi_{h}$. Hence we can regard $C\left(\mathbb{G}_{\text {red }}\right)=\pi_{h}(C(\mathbb{G}))$. We often omit $\pi_{h}$.

2.3. Multiplicative unitaries. From the bi-invariance of the state $h$, the following theorem follows:

THEOREM 2.5. There exists a unitary $V \in B\left(L^{2}(\mathbb{G}) \otimes L^{2}(\mathbb{G})\right)$ satisfying

$$
V\left(a \Omega_{h} \otimes \xi\right)=\delta(a)\left(\Omega_{h} \otimes \xi\right) \text { for all } a \in C(\mathbb{G}), \xi \in L^{2}(\mathbb{G}) ;
$$

Then $V$ satisfies the following notable pentagon equation:

$$
V_{12} V_{13} V_{23}=V_{23} V_{12} \text {. }
$$

So, $V$ is called the multiplicative unitary [5]. By definition, we have the following implementation formula:

$$
V(a \otimes 1) V^{*}=\delta(a) \quad \text { for all } a \in C\left(\mathbb{G}_{\mathrm{red}}\right) .
$$

2.4. Von Neumann algebraic quantum groups. We denote by $L^{\infty}(\mathbb{G})$ the weak closure of $C\left(\mathbb{G}_{\text {red }}\right)$ in $B\left(L^{2}(\mathbb{G})\right)$. The coproduct $\delta$ extends to the normal morphism from $L^{\infty}(\mathbb{G})$ into $L^{\infty}(\mathbb{G}) \otimes L^{\infty}(\mathbb{G})$ through 2.2$)$. Then the pair $\left(L^{\infty}(\mathbb{G}), \delta\right)$ is called the von Neumann algebraic compact quantum group [11] VT. There exists a modular automorphism for $h$ on $C\left(\mathbb{G}_{\text {red }}\right)$, and the Haar state $h(\cdot)=\left(\cdot \Omega_{h}, \Omega_{h}\right)$ is faithful on $L^{\infty}(\mathbb{G})[22$.

\subsection{Kac type quantum groups}

Definition 2.6. A compact quantum group is said to be of Kac type when the Haar state is tracial, i.e. $h(a b)=h(b a)$ for all $a, b \in C(\mathbb{G})$.

A compact group or a $C^{*}$-group algebra of a discrete group are typical examples of Kac type quantum groups. They are commutative or co-commutative. Woronowicz's twisted quantum group $S U_{-1}(n)$ is also of Kac type, which is neither commutative nor co-commutative 21. Readers should note the first such example discovered by G. I. Kac and V. G. Paljutkin [9], which is 8 -dimensional $C^{*}$-algebra (8 is the smallest dimension allowing a non-trivial Kac algebra).

\subsection{Representation theory}

Definition 2.7. Let $H$ be a Hilbert space. A unitary $v \in B(H) \otimes L^{\infty}(\mathbb{G})$ is called a (right unitary) representation if it satisfies

$$
(\mathrm{id} \otimes \delta)(v)=v_{12} v_{13} .
$$

We have the following natural operations:

- (direct sum)

$$
v_{1} \oplus v_{2}:=\left(\begin{array}{cc}
v_{1} & 0 \\
0 & v_{2}
\end{array}\right) \in B\left(H_{1} \oplus H_{2}\right) \otimes L^{\infty}(\mathbb{G}) .
$$


- (tensor product)

$$
v_{1} \otimes v_{2}:=\left(v_{1}\right)_{13}\left(v_{2}\right)_{23} \in B\left(H_{1} \otimes H_{2}\right) \otimes L^{\infty}(\mathbb{G}) .
$$

- (conjugation) Let $v=\left(v_{i j}\right)_{i, j \in I}$ be a matrix form of a representation. Consider

$$
v^{c}:=\left(v_{i j}^{*}\right)_{i, j \in I}
$$

which may not be a unitary, but this still satisfies 2.3 . In fact, if the dimension is finite, then it is unitarizable, i.e. there exists a positive invertible operator $Q$ such that

$$
\bar{v}:=\left(Q^{1 / 2} \otimes 1\right) v^{c}\left(Q^{-1 / 2} \otimes 1\right)
$$

is a unitary matrix (see [22]).

We introduce the intertwiner space between unitary representations $v_{i} \in B\left(H_{i}\right) \otimes$ $L^{\infty}(\mathbb{G}), i=1,2$,

$$
\operatorname{Mor}\left(v_{1}, v_{2}\right):=\left\{T \in B\left(H_{1}, H_{2}\right) \mid(T \otimes 1) v_{1}=v_{2}(T \otimes 1)\right\} .
$$

Definition 2.8. Let $v \in B(H) \otimes L^{\infty}(\mathbb{G})$ be a unitary representation.

- A unitary representation is said to be irreducible when $\operatorname{Mor}(v, v)=\mathbb{C} 1_{H}$.

- Let $w \in B(K) \otimes L^{\infty}(\mathbb{G})$ be a unitary representation. We say that $v$ and $w$ are equivalent if $\operatorname{Mor}(v, w)$ contains a unitary.

THEOREM 2.9 (Woronowicz). For any compact quantum group $\mathbb{G}$, the following hold:

1. An irreducible representation is finite dimensional;

2. A finite dimensional representation is the direct sum of irreducibles;

3. Let $v \in B(H) \otimes L^{\infty}(\mathbb{G})$ be a finite dimensional representation. Then $v \in B(H) \otimes$ $C(\mathbb{G})$.

We denote by $\operatorname{Irr}(\mathbb{G})$ the set of equivalence classes of irreducible representations of $\mathbb{G}$. For each $\pi \in \operatorname{Irr}(\mathbb{G})$, we choose a corresponding representation $v_{\pi} \in B\left(H_{\pi}\right) \otimes L^{\infty}(\mathbb{G})$. Note that $\operatorname{dim}\left(H_{\pi}\right)<\infty$ from the previous theorem.

Definition 2.10. We say that $\mathbb{G}$ has commutative fusion rules when $v \otimes w$ is equivalent to $w \otimes v$ for any unitary representations $v, w$.

EXAMPLE 2.11. If $\mathbb{G}$ is a compact group, then a usual flip map intertwines $v \otimes w$ and $w \otimes v$. For a $q$-deformation, an $R$-matrix takes place of that.

3. Discrete quantum groups. Let $\mathbb{G}$ be a c.q.g. In this section, we study basic properties of the dual $\widehat{\mathbb{G}}$.

3.1. Right group algebras. Recall the multiplicative unitaries $V$, which are right and left representations of $\mathbb{G}$ on $L^{2}(\mathbb{G})$. We introduce the following subspace:

$$
R(\mathbb{G}):=\overline{\operatorname{span}}^{\mathrm{w}}\left\{(\mathrm{id} \otimes \omega)(V) \mid \omega \in L^{\infty}(\mathbb{G})_{*}\right\},
$$

which we call the right group algebra.

Define the map $\beta: B\left(L^{2}(\mathbb{G})\right) \rightarrow B\left(L^{2}(\mathbb{G})\right) \otimes B\left(L^{2}(\mathbb{G})\right)$,

$$
\beta(x):=V^{*}(1 \otimes x) V \text { for } x \in B\left(L^{2}(\mathbb{G})\right) .
$$


THEOREM 3.1. The following hold:

- $R(\mathbb{G})$ is a von Neumann algebra;

- The restriction $\Delta:=\left.\beta\right|_{R(\mathbb{G})}$ defines the coproduct, i.e.

$$
\Delta(R(\mathbb{G})) \subset R(\mathbb{G}) \otimes R(\mathbb{G}), \quad(\Delta \otimes \mathrm{id}) \circ \Delta=(\mathrm{id} \otimes \Delta) \circ \Delta
$$

So, the pair $(R(\mathbb{G}), \Delta)$ is a bialgebra. In fact, it is known that there exist weights $\varphi$ and $\psi$ on $R(\mathbb{G})$ such that

- $\varphi((\omega \otimes \mathrm{id})(\Delta(x)))=\omega(1) \psi(x)$ for all $\omega \in R(\mathbb{G})_{*}^{+}, x \in R(\mathbb{G})_{+}$.

- $\psi((\operatorname{id} \otimes \omega)(\Delta(x)))=\omega(1) \varphi(x)$ for all $\omega \in R(\mathbb{G})_{*}^{+}, x \in R(\mathbb{G})_{+}$;

Therefore, $\widehat{\mathbb{G}}:=(R(\mathbb{G}), \Delta)$ is a quantum group in the sense of [1]. Using the usual Peter-Weyl type isomorphism,

$$
L^{2}(\mathbb{G}) \cong \bigoplus_{\pi \in \operatorname{Irr}(\mathbb{G})} H_{\bar{\pi}} \otimes H_{\pi}
$$

we obtain the isomorphism,

$$
R(\mathbb{G}) \rightarrow \bigoplus_{\pi \in \operatorname{Irr}(\mathbb{G})} B\left(H_{\pi}\right)
$$

Hence $\widehat{\mathbb{G}}$ is also called a discrete quantum group.

\subsection{Actions of quantum groups}

Definition 3.2. Let $\mathbb{G}=\left(L^{\infty}(\mathbb{G}), \delta\right)$ be a locally compact quantum group and $M$ a von Neumann algebra. A map $\alpha: M \rightarrow M \otimes L^{\infty}(\mathbb{G})$ is called a (right) action when it satisfies the following:

- $\alpha$ is a unital faithful normal $*$-homomorphism;

- $($ id $\otimes \delta) \circ \alpha=(\alpha \otimes$ id $) \circ \alpha$.

A left action is similarly defined.

EXAmple 3.3. The map $\beta: B\left(L^{2}(\mathbb{G})\right) \rightarrow R(\mathbb{G}) \otimes B\left(L^{2}(\mathbb{G})\right)$ is a left action of $\widehat{\mathbb{G}}$. Similarly $\alpha: B\left(L^{2}(\mathbb{G})\right) \rightarrow B\left(L^{2}(\mathbb{G})\right) \otimes L^{\infty}(\mathbb{G})$ defined by $\alpha(x)=V(x \otimes 1) V^{*}$ is a right action of $\mathbb{G}$.

3.3. Quantum subgroups and left (right) coideals. There are several ways to define a quantum subgroup of a compact quantum group, but we adopt the following definition in this article.

Definition 3.4. Let $\mathbb{G}$ and $\mathbb{H}$ be compact quantum groups. We say that $\mathbb{H}$ is a quantum subgroup of $\mathbb{G}$ if there exists a unital $*$-homomorphism $r_{\mathbb{H}}: A(\mathbb{G}) \rightarrow A(\mathbb{H})$ such that

- $r_{\mathbb{H}}$ is surjective;

- $\delta_{\mathbb{H}} \circ r_{\mathbb{H}}=\left(r_{\mathbb{H}} \otimes r_{\mathbb{H}}\right) \circ \delta_{\mathbb{G}}$.

This definition is weaker than the usual $C^{*}$-version, which requires $r_{\mathbb{H}}$ is a $C^{*}$ homomorphism from $C(\mathbb{G})$ onto $C(\mathbb{H})$. Readers should notice that the map $r_{\mathbb{H}}$, called the restriction, need not be unique. So, rigorously we have to say the pair $\left\{\mathbb{H}, r_{\mathbb{H}}\right\}$ is a 
quantum subgroup, but we simply say $\mathbb{H}$ is a quantum subgroup. Note that $\mathbb{H}$ acts on $A(\mathbb{G})$ from both sides as $\mathbb{H} \stackrel{\gamma^{\ell}}{\curvearrowright} A(\mathbb{G}) \stackrel{\gamma^{r}}{\curvearrowleft} \mathbb{H}$ defined by

$$
\gamma^{\ell}:=\left(r_{\mathbb{H}} \otimes \mathrm{id}\right) \circ \delta_{\mathbb{G}}, \quad \gamma^{r}:=\left(\mathrm{id} \otimes r_{\mathbb{H}}\right) \circ \delta_{\mathbb{G}} .
$$

Then we define the non-commutative quotient spaces by the following fixed point algebras:

$$
\begin{aligned}
& A(\mathbb{H} \backslash \mathbb{G}):=\left\{a \in A(\mathbb{G}) \mid \gamma^{\ell}(a)=1 \otimes a\right\}, \\
& A(\mathbb{G} / \mathbb{H}):=\left\{a \in A(\mathbb{G}) \mid \gamma^{r}(a)=a \otimes 1\right\} .
\end{aligned}
$$

The weak closures in $B\left(L^{2}(\mathbb{G})\right)$ are denoted by $L^{\infty}(\mathbb{H} \backslash \mathbb{G})$ and $L^{\infty}(\mathbb{G} / \mathbb{H})$, respectively.

Note that the left $\mathbb{H}$-action $\gamma^{\ell}$ and the right $\mathbb{G}$-action $\delta_{\mathbb{G}}$ are commuting, i.e. $\left(\right.$ id $\left.\otimes \delta_{\mathbb{G}}\right) \circ$ $\gamma^{\ell}=\left(\gamma^{\ell} \otimes \mathrm{id}\right) \circ \delta_{\mathbb{G}}$. Hence $\mathbb{G}$ is also acting on $A(\mathbb{H} \backslash \mathbb{G})$ by $\delta$. Similarly the coproduct $\delta_{\mathbb{G}}$ defines a left action on $A(\mathbb{G} / \mathbb{H})$. Since these actions preserve the Haar state, they extend to the quotient spaces $L^{\infty}(\mathbb{H} \backslash \mathbb{G})$ or $L^{\infty}(\mathbb{G} / \mathbb{H})$, respectively. They are typical examples of right or left coideals.

Definition 3.5. Let $B \subset L^{\infty}(\mathbb{G})$ be a von Neumann subalgebra. Then we say that

- $B$ is a left coideal if $\delta(B) \subset L^{\infty}(\mathbb{G}) \otimes B$;

- $B$ is a right coideal if $\delta(B) \subset B \otimes L^{\infty}(\mathbb{G})$;

- a left (right) coideal $B$ is of quotient type if $B=L^{\infty}(\mathbb{G} / \mathbb{H})\left(\operatorname{resp} . L^{\infty}(\mathbb{H} \backslash \mathbb{G})\right.$ ) for some quantum subgroup $\mathbb{H}$.

Thanks to Gelfand theorem, every left coideal is of quotient type when $\mathbb{G}$ is a compact group [1. However, this is not true in general [13, 14, 17]. Indeed, we have the following characterization $[18$.

Theorem 3.6 (Tomatsu). Let $B \subset L^{\infty}(\mathbb{G})$ be a right coideal. Then the following are equivalent:

- $B$ is of quotient type;

- There exists an expectation $E_{B}: L^{\infty}(\mathbb{G}) \rightarrow B$ preserving the Haar state, and moreover $\widehat{\mathbb{G}}$ acts on $B$, i.e. $\beta(B) \subset R(\mathbb{G}) \otimes B$.

This theorem has been proved for co-amenable quantum groups [18, but the same proof works because we have changed the definition of quantum subgroups.

3.4. Amenability and co-amenability. For details of the theory of amenability for quantum groups, readers are referred to [2, 3, 4, 16] and references therein.

DeFinition 3.7. We say that $\widehat{\mathbb{G}}$ is amenable when there exists an invariant mean $m$ on $R(\mathbb{G})$, that is, $m \in R(\mathbb{G})^{*}$ is a state such that

$$
m((\mathrm{id} \otimes \omega)(\Delta(x)))=\omega(1) m(x)=m((\omega \otimes \mathrm{id})(\Delta(x))) .
$$

In this case, $\mathbb{G}$ is said to be co-amenable.

Theorem 3.8 (Bedos-Murphy-Tuset, Tomatsu). The following are equivalent:

- $\mathbb{G}$ is co-amenable;

- $C\left(\mathbb{G}_{\mathrm{red}}\right)$ has a bounded counit $\varepsilon$ that is a *homomorphism $\varepsilon: C\left(\mathbb{G}_{\mathrm{red}}\right) \rightarrow \mathbb{C}$ such that $(\varepsilon \otimes \mathrm{id}) \circ \delta=\mathrm{id}=(\mathrm{id} \otimes \varepsilon) \circ \delta$. 
4. Poisson boundaries. We briefly recall the notion of the Poisson boundary for a discrete quantum group. We refer to [7, 8] for definitions of terminology.

4.1. Identification problems. Let $\phi_{\pi} \in B\left(H_{\pi}\right)_{*}$ be a right $\mathbb{G}$-invariant state. Define a transition operator $P_{\pi}$ on $R(\mathbb{G})$ by $P_{\pi}(x)=\left(\mathrm{id} \otimes \phi_{\pi}\right)\left(\Delta_{R}(x)\right)$ for $x \in R(\mathbb{G})$. When $\widehat{\mathbb{G}}$ is a discrete group, $P_{g}, g \in \widehat{\mathbb{G}}$, is nothing but the right translation of functions by $g \in \widehat{\mathbb{G}}$, which is an automorphism. However, the map $P_{\pi}$ is not an automorphism but a faithful normal unital completely positive (u.c.p.) map in general.

For a probability measure $\mu$ on $\operatorname{Irr}(\mathbb{G})$, we set the non-commutative Markov operator,

$$
P_{\mu}:=\sum_{\pi \in \operatorname{Irr}(\mathbb{G})} \mu(\pi) P_{\pi} .
$$

We assume $\mu$ is generating, that is, $\operatorname{supp}(\mu)$ generates $\operatorname{Irr}(\mathbb{G})$ as a semigroup in the following sense: for any $\pi \in \operatorname{Irr}(\mathbb{G})$, there exist $\rho_{1}, \ldots, \rho_{n} \in \operatorname{supp}(\mu)$ such that the representation $\pi$ is contained in the tensor product representation $\rho_{1} \otimes \cdots \otimes \rho_{n}$.

Then we define an operator system,

$$
H^{\infty}\left(\widehat{\mathbb{G}}, P_{\mu}\right):=\left\{x \in R(\mathbb{G}) \mid P_{\mu}(x)=x\right\} .
$$

We often regard id $-P_{\mu}$ as a Laplace operator on $\widehat{\mathbb{G}}$, and we say that each element of $H^{\infty}\left(\widehat{\mathbb{G}}, P_{\mu}\right)$ is $P_{\mu}$-harmonic. That operator system has the von Neumann algebra structure defined by

$$
x \cdot y=\lim _{n \rightarrow \infty} P_{\mu}^{n}(x y) \quad \text { for } x, y \in H^{\infty}\left(\widehat{\mathbb{G}}, P_{\mu}\right),
$$

where the limit is taken in the strong topology [7, Theorem 3.6]. The von Neumann algebra $H^{\infty}\left(\widehat{\mathbb{G}}, P_{\mu}\right)$ is called the (non-commutative) Poisson boundary of $\left\{R(\mathbb{G}), P_{\mu}\right\}$. The following theorem is probably well-known to specialists.

TheOREM 4.1. The von Neumann algebra $H^{\infty}\left(\widehat{\mathbb{G}}, P_{\mu}\right)$ is amenable.

Proof. We know that $H^{\infty}\left(\widehat{\mathbb{G}}, P_{\mu}\right)$ is isomorphic to $\left(\mathcal{R}^{\alpha}\right)^{\prime} \cap \mathcal{R}$, where $\mathcal{R} \stackrel{\alpha}{\curvearrowleft} \mathbb{G}$ is an ITP action [7. Let $E_{\alpha}:=($ id $\otimes h) \circ \alpha$ be the averaging expectation. Take a faithful state $\omega \in\left(\mathcal{R}^{\alpha}\right)_{*}$, and set $\psi:=\omega \circ E_{\alpha}$. Since $\left.\sigma_{t}^{\psi}\right|_{\mathcal{R}^{\alpha}}=\sigma_{t}^{\omega}, \sigma_{t}^{\psi}\left(\left(\mathcal{R}^{\alpha}\right)^{\prime} \cap \mathcal{R}\right)=\left(\mathcal{R}^{\alpha}\right)^{\prime} \cap \mathcal{R}$. Thanks to Takesaki theorem [15], we see that there exists an expectation from $\mathcal{R}$ onto $\left(\mathcal{R}^{\mathbb{G}}\right)^{\prime} \cap \mathcal{R}$, and $\left(\mathcal{R}^{\mathbb{G}}\right)^{\prime} \cap \mathcal{R}$ is amenable. Hence so is $H^{\infty}\left(\widehat{\mathbb{G}}, P_{\mu}\right)$.

Now we recall the actions $\widehat{\mathbb{G}} \stackrel{\beta}{\curvearrowright} B\left(L^{2}(\mathbb{G})\right) \stackrel{\alpha}{\curvearrowleft} \mathbb{G}$ defined by

$$
\beta(x):=V^{*}(1 \otimes x) V, \quad \alpha(x)=V(x \otimes 1) V^{*} .
$$

Since we can prove $P_{\mu}$ and $\alpha$ or $\beta$ are commuting on $R(\mathbb{G})$, the Poisson boundary is a $\widehat{\mathbb{G}}-\mathbb{G}$-von Neumann algebra [7]. We should note that if $\widehat{\mathbb{G}}$ is a discrete group, then $\alpha$ is trivial. Hence non-triviality of $\alpha$ on $R(\mathbb{G})$ is a purely quantum phenomenon.

In Poisson boundary theory, one of the most important problems is the following:

Problem 4.2 (Identification problem). Realize $H^{\infty}\left(\widehat{\mathbb{G}}, P_{\mu}\right)$ as a more concrete $\widehat{\mathbb{G}}-\mathbb{G}$-von Neumann algebra.

In [7], it is shown that $H^{\infty}\left(S U_{q}(2), P_{\mu}\right) \cong L^{\infty}\left(\mathbb{T} \backslash S U_{q}(2)\right)$. This result is generalized to $S U_{q}(n)$ by Izumi-Neshveyev-Tuset [8]. Their key ingredient is the Poisson integral 
$\Theta: L^{\infty}(\mathbb{G}) \rightarrow R(\mathbb{G})$ defined by

$$
\Theta(a):=(\operatorname{id} \otimes h)(\beta(a)) \text { for } a \in L^{\infty}(\mathbb{G}) .
$$

Then the faithful normal u.c.p. map $\Theta$ is $\widehat{\mathbb{G}}-\mathbb{G}$-equivariant, i.e.

$$
\Delta \circ \Theta=(\mathrm{id} \otimes \Theta) \circ \beta, \quad \alpha \circ \Theta=(\Theta \otimes \mathrm{id}) \circ \delta .
$$

It is known that the range space $\operatorname{Im} \Theta$ is contained in $H^{\infty}\left(\widehat{\mathbb{G}}, P_{\mu}\right)$. Here, we should note that $\operatorname{Im} \Theta$ does not depend on $\mu$. Indeed, if we define the operator system

$$
H^{\infty}(\widehat{\mathbb{G}}):=\left\{x \in R(\mathbb{G}) \mid P_{\pi}(x)=x \text { for all } \pi \in \operatorname{Irr}(\mathbb{G})\right\},
$$

then $\operatorname{Im} \Theta \subset H^{\infty}(\widehat{\mathbb{G}})$.

From now, we focus on $\mathbb{G}$ with commutative fusion rules. Then $H^{\infty}\left(\widehat{\mathbb{G}}, P_{\mu}\right)=H^{\infty}(\widehat{\mathbb{G}})$ for any generating probability measure $\mu[8]$.

Our main theorem of this section is the following [18]:

THEOREM 4.3. Let $\mathbb{G}$ be a co-amenable compact quantum group. Assume that its fusion algebra is commutative. Then the following statements hold:

1. There exists a unique maximal quantum subgroup of Kac type $\mathbb{H}$, that is, if $\mathbb{K}$ is another Kac quantum subgroup of $\mathbb{G}$, then $L^{\infty}(\mathbb{H} \backslash \mathbb{G}) \subset L^{\infty}(\mathbb{K} \backslash \mathbb{G})$;

2. The Poisson integral $\Theta: L^{\infty}(\mathbb{H} \backslash \mathbb{G}) \rightarrow H^{\infty}(\widehat{\mathbb{G}})$ is an isomorphism.

Proof. We present a sketch of a proof. What we want to construct first is the inverse map $\Lambda: H^{\infty}(\widehat{\mathbb{G}}) \rightarrow L^{\infty}(\mathbb{G})$. To do this, we recall $\Theta=($ id $\otimes h) \circ \beta$. Then we would use a "Haar state" on $R(\mathbb{G})$, intuitively. If $\widehat{\mathbb{G}}$ was finite dimensional, it would be sufficient to replace $\beta$ with $\alpha$. A problem is that we do not have such a functional in general. However, since we have assumed amenability of $\widehat{\mathbb{G}}$, we have an invariant mean $m$ on $R(\mathbb{G})$, which may be singular. Then we introduce $\Lambda: R(\mathbb{G}) \rightarrow L^{\infty}(\mathbb{G})$ given by

$$
\Lambda(x):=(m \otimes \mathrm{id})(\alpha(x)) \text { for } x \in R(\mathbb{G}),
$$

where the u.c.p. map $m \otimes \mathrm{id}: R(\mathbb{G}) \otimes L^{\infty}(\mathbb{G}) \rightarrow L^{\infty}(\mathbb{G})$ is well-defined.

Next we compute $\Theta \circ \Lambda$, and we obtain $\Theta \circ \Lambda=$ id on $H^{\infty}(\widehat{\mathbb{G}})$ because of the ergodicity of $H^{\infty}(\widehat{\mathbb{G}}) \stackrel{\alpha}{\curvearrowleft} \mathbb{G}$, which comes from the commutativity of fusion rules. In particular, we have shown $\operatorname{Im} \Theta=H^{\infty}(\widehat{\mathbb{G}})$. Moreover, we can see that $\Lambda$ is multiplicative on $H^{\infty}(\widehat{\mathbb{G}})$ : for any $x \in H^{\infty}(\widehat{\mathbb{G}})$, we have

$$
x^{*} \cdot x=\Theta(\Lambda(x))^{*} \cdot \Theta(\Lambda(x)) \leq \Theta\left(\Lambda(x)^{*} \Lambda(x)\right) \leq \Theta\left(\Lambda\left(x^{*} \cdot x\right)\right)=x^{*} \cdot x .
$$

Hence we obtain $\Theta\left(\Lambda(x)^{*} \Lambda(x)\right)=\Theta\left(\Lambda\left(x^{*} \cdot x\right)\right)$. Since $\Lambda(x)^{*} \Lambda(x) \leq \Lambda\left(x^{*} \cdot x\right)$ and $\Theta$ is faithful, $\Lambda(x)^{*} \Lambda(x)=\Lambda\left(x^{*} \cdot x\right)$ holds.

Next we study the image of $\Lambda$, and put $B:=\operatorname{Im} \Lambda$, which is a von Neumann subalgebra in $L^{\infty}(\mathbb{G})$. We consider $E:=\Lambda \circ \Theta: L^{\infty}(\mathbb{G}) \rightarrow L^{\infty}(\mathbb{G})$. It is easy to see that $E$ is a Haar state preserving expectation onto $B$. Since $\Lambda$ is $\widehat{\mathbb{G}}-\mathbb{G}$-equivariant, $\widehat{\mathbb{G}}$ and $\mathbb{G}$ are acting on $B$. Hence $B$ is a right coideal with nice properties, and $B$ must be of quotient type by Theorem 3.6 .

So, $B$ is of the form $L^{\infty}(\mathbb{H} \backslash \mathbb{G})$ for some quantum subgroup $\mathbb{H}$. By definition, $E$ is also $\widehat{\mathbb{G}}$-equivariant, and we see that $\mathbb{H}$ is of Kac type. With a little effort, we can see that $\mathbb{H}$ is the maximal quantum subgroup of Kac type. 
For the $q$-deformation of a classical compact Lie group, we can show that the maximal Kac quantum subgroup is exactly equal to the maximal torus [18. Hence we have the following result which generalizes the main result of $[8]$ :

Corollary 4.4. Let $\mathbb{G}_{q}$ be the $q$-deformation of a classical compact Lie group $\mathbb{G}$. Then the Poisson integral $\Theta: L^{\infty}\left(\mathbb{T} \backslash \mathbb{G}_{q}\right) \rightarrow H^{\infty}\left(\widehat{\mathbb{G}_{q}}\right)$ is an isomorphism.

4.2. Description of centers of Poisson boundaries. Here, we will propose a new problem on a Poisson boundary. Recall that $P_{\mu}$ and $\alpha$ are commuting, and $P_{\mu}$ acts on the fixed point algebra $R(\mathbb{G})^{\alpha}$, which is nothing but the center $Z(R(\mathbb{G}))=\ell_{\infty}(\operatorname{Irr}(\mathbb{G}))$. Hence we introduce the classical part of a Poisson boundary,

$$
H^{\infty}\left(\widehat{\mathbb{G}}, P_{\mu}\right)_{\text {class }}:=H^{\infty}\left(\widehat{\mathbb{G}}, P_{\mu}\right) \cap Z(R(\mathbb{G})) .
$$

Let us denote the center of $H^{\infty}\left(\widehat{\mathbb{G}}, P_{\mu}\right)$ by $Z\left(H^{\infty}\left(\widehat{\mathbb{G}}, P_{\mu}\right)\right)$, where we should again remember the product structure 4.1. It is trivial by 4.1 that the classical part $H^{\infty}\left(\widehat{\mathbb{G}}, P_{\mu}\right)_{\text {class }}$ is contained in $Z\left(H^{\infty}\left(\widehat{\mathbb{G}}, P_{\mu}\right)\right)$. Now we present following our problem:

CONJECTURE 4.5. Let $\mathbb{G}$ be a compact quantum group and $\mu$ a generating probability measure. Then the following equality holds:

$$
H^{\infty}\left(\widehat{\mathbb{G}}, P_{\mu}\right)_{\text {class }}=Z\left(H^{\infty}\left(\widehat{\mathbb{G}}, P_{\mu}\right)\right) .
$$

When $\mathbb{G}$ has commutative fusion rules, then the classical part is trivial [6, 7]. So, the conjecture means the factoriality of $H^{\infty}\left(\widehat{\mathbb{G}}, P_{\mu}\right)$.

There are some positive observations about this conjecture. For $S U_{q}(2)$ case, it is true because the quantum flag manifold (or a Podleś sphere) $L^{\infty}\left(\mathbb{T} \backslash S U_{q}(2)\right.$ ) is a type $\mathrm{I}_{\infty}$ factor. However, that is unknown for other $q$-deformations. The conjecture holds even for non-amenable examples such as $A_{o}(F)$ and $A_{u}(F)$ [19, 20]. It has seemed to be affirmative so far.

4.3. A criterion on a factoriality of $L^{\infty}\left(\mathbb{T}_{\max } \backslash \mathbb{G}\right)$. From now on, $\mathbb{G}$ is the $q$-deformation of a classical compact Lie group [10. It is proved that the maximal Kac quantum subgroup of $\mathbb{G}$ is the maximal torus $\mathbb{T}_{\max }[18$. Then Theorem 4.3 implies the isomorphism,

$$
\Theta: L^{\infty}\left(\mathbb{T}_{\max } \backslash \mathbb{G}\right) \rightarrow H^{\infty}(\widehat{\mathbb{G}}) .
$$

Recall the left action $\mathbb{T}_{\max } \stackrel{\gamma^{\ell}}{\curvearrowright} L^{\infty}(\mathbb{G})$ introduced in $\$ 3.3$.

LEMmA 4.6. Let $Z$ be the center of $L^{\infty}(\mathbb{G})$. Then the left action $\mathbb{T}_{\max } \stackrel{\gamma^{\ell}}{\curvearrowright} L^{\infty}(\mathbb{G})$ is centrally ergodic, i.e. $Z^{\gamma^{\ell}}=\mathbb{C}$

Proof. Recall the left $\widehat{\mathbb{G}}$-action $\beta(x)=V^{*}(x \otimes 1) V, x \in L^{\infty}(\mathbb{G})$. Then $L^{\infty}(\mathbb{G})^{\beta}=Z$. Since $Z^{\gamma^{\ell}}=L^{\infty}\left(\mathbb{T}_{\max } \backslash \mathbb{G}\right)^{\beta}, \Theta\left(Z^{\gamma^{\ell}}\right)=H^{\infty}(\widehat{\mathbb{G}})^{\beta}$, this is equal to $\mathbb{C}$ because $R(\mathbb{G})^{\beta}=\mathbb{C}$. Hence $Z^{\gamma^{\ell}}=\Theta^{-1}(\mathbb{C})=\mathbb{C}$.

Hence there exists a subgroup $\Gamma \subset \widehat{\mathbb{T}_{\max }}$ such that the following decomposition holds:

$$
Z=\bigoplus_{\chi \in \Gamma} Z_{\chi}
$$

where $Z_{\chi}:=\left\{x \in Z \mid \gamma_{z}^{\ell}(x)=\chi(z) x\right.$ for $\left.z \in \mathbb{T}_{\max }\right\}$. From the previous lemma, $Z_{0}=\mathbb{C}$, and each $Z_{\chi}$ is one-dimensional subspace spanned by a unitary. 
THEOREM 4.7. If the action $\mathbb{T}_{\max } \stackrel{\gamma^{\ell}}{\curvearrowright} Z$ is faithful, then the following hold:

1. $L^{\infty}(\mathbb{G})=Z \vee L^{\infty}\left(\mathbb{T}_{\max } \backslash \mathbb{G}\right)$;

2. $L^{\infty}\left(\mathbb{T}_{\max } \backslash \mathbb{G}\right)$ is the type $I_{\infty}$ factor;

3. $H^{\infty}(\widehat{\mathbb{G}})$ is the type $I_{\infty}$ factor.

Proof. By our assumption, $\Gamma$ coincides with $\widehat{\mathbb{T}_{\max }}$. Hence for any $\chi \in \widehat{\mathbb{T}_{\max }}$, there exists a unitary $u_{\chi} \in Z_{\chi}$. Then $u_{\chi}^{*} L^{\infty}(\mathbb{G})_{\chi}=L^{\infty}(\mathbb{G})^{\gamma^{\ell}}=L^{\infty}\left(\mathbb{T}_{\max } \backslash \mathbb{G}\right)$. Hence $L^{\infty}(\mathbb{G})_{\chi}=$ $u_{\chi} L^{\infty}\left(\mathbb{T}_{\max } \backslash \mathbb{G}\right)$, and (1) holds.

It is known that $C(\mathbb{G})$ is a type I $C^{*}$-algebra $\left[10\right.$, and so are $L^{\infty}(\mathbb{G})$ and $L^{\infty}\left(\mathbb{T}_{\max } \backslash \mathbb{G}\right)$, which is trivially infinite dimensional. By $(1)$, the center of $L^{\infty}\left(\mathbb{T}_{\max } \backslash \mathbb{G}\right)$ is contained in $Z$. The central ergodicity of $\gamma^{\ell}$ implies the factoriality of $L^{\infty}\left(\mathbb{T}_{\max } \backslash \mathbb{G}\right)$.

(3) is trivial from Theorem 4.3 .

From the next subsection, we present a complete proof for the $S U_{q}(2)$ case.

4.4. Quantum group $S U_{q}(2)$. The definition of $S U_{q}(2)$ is as follows. Our notations are same as those of [12. The continuous function algebra $C\left(S U_{q}(2)\right)$ is the universal $C^{*}$-algebra generated by four elements $x, u, v$ and $y$ with the following relations:

$$
\begin{gathered}
u x=q x u, \quad v x=q x v, \quad y u=q u y, \quad y v=q v y, \quad u v=v u, \\
x y-q^{-1} u v=1=y x-q u v, \\
x^{*}=y, \quad u^{*}=-q^{-1} v .
\end{gathered}
$$

The coproduct $\delta$ is given by

$$
\left(\begin{array}{ll}
\delta(x) & \delta(u) \\
\delta(v) & \delta(y)
\end{array}\right):=\left(\begin{array}{ll}
x \otimes 1 & u \otimes 1 \\
v \otimes 1 & y \otimes 1
\end{array}\right) \cdot\left(\begin{array}{ll}
1 \otimes x & 1 \otimes u \\
1 \otimes v & 1 \otimes y
\end{array}\right),
$$

which means the following matrix is an (irreducible) representation:

$$
w(1 / 2):=\left(\begin{array}{ll}
x & u \\
v & y
\end{array}\right) .
$$

Each element of $\operatorname{Irr}\left(S U_{q}(2)\right)$ is determined by the highest weight, which is an element of $(1 / 2) \mathbb{Z}_{+}=\{0,1 / 2, \cdots\}$ in this case. Each $\nu \in(1 / 2) \mathbb{Z}_{+}$is called the spin and the dimension of $w(\nu)$ is $2 \nu+1$. The quantum dimension of $w(\nu)$ is given by the $q$-integer $(2 \nu+1)_{q}:=\left(q^{-2 \nu-1}-q^{2 \nu+1}\right) /\left(q^{-1}-q\right)[12$.

On tensor products, we have the same formula (Clebsch-Gordan rule) as that of $S U(2)$,

$$
w(\mu) \oplus w(\nu)=w(|\mu-\nu|) \oplus w(|\mu-\nu|+1) \oplus \cdots \oplus w(\mu+\nu-1) \oplus w(\mu+\nu) .
$$

For $\ell \in(1 / 2) \mathbb{Z}_{+}$, we set $I_{\ell}:=\{-\ell,-\ell+1, \ldots, \ell-1, \ell\}$. Set the positive operator $\zeta:=u^{*} u=-q^{-1} u v$.

THEOREM 4.8. For each $\ell \in(1 / 2) \mathbb{Z}_{+}$and $i, j \in I_{\ell}$, the matrix elements $w(\ell)_{i, j}$ are expressed in terms of the little q-Jacobi polynomials in $\zeta$ as follows:

1. Case $i+j \leq 0, i \geq j$ :

$$
q^{(\ell+j)(j-i)}\left[\begin{array}{l}
\ell+i \\
i-j
\end{array}\right]_{q^{2}}^{\frac{1}{2}}\left[\begin{array}{l}
\ell-j \\
i-j
\end{array}\right]_{q^{2}}^{\frac{1}{2}} x^{-i-j} v^{i-j} P_{\ell+j}^{(i-j,-i-j)}\left(\zeta ; q^{2}\right)
$$


2. Case $i+j \leq 0, i \leq j$ :

$$
q^{(\ell+i)(i-j)}\left[\begin{array}{l}
\ell-i \\
j-i
\end{array}\right]_{q^{2}}^{\frac{1}{2}}\left[\begin{array}{c}
\ell+j \\
j-i
\end{array}\right]_{q^{2}}^{\frac{1}{2}} x^{-i-j} u^{j-i} P_{\ell+i}^{(j-i,-i-j)}\left(\zeta ; q^{2}\right)
$$

3. Case $i+j \geq 0, i \leq j$ :

$$
q^{(j-i)(j-\ell)}\left[\begin{array}{l}
\ell-i \\
j-i
\end{array}\right]_{q^{2}}^{\frac{1}{2}}\left[\begin{array}{c}
\ell+j \\
j-i
\end{array}\right]_{q^{2}}^{\frac{1}{2}} P_{\ell-j}^{(j-i, i+j)}\left(\zeta ; q^{2}\right) u^{j-i} y^{i+j}
$$

4. Case $i+j \geq 0, i \geq j$ :

$$
q^{(i-j)(i-\ell)}\left[\begin{array}{l}
\ell+i \\
i-j
\end{array}\right]_{q^{2}}^{\frac{1}{2}}\left[\begin{array}{l}
\ell-j \\
i-j
\end{array}\right]_{q^{2}}^{\frac{1}{2}} P_{\ell-i}^{(i-j, i+j)}\left(\zeta ; q^{2}\right) v^{i-j} y^{i+j},
$$

where we have used the q-binomial coefficients and the little q-Jacobi polynomials:

$$
\begin{gathered}
{\left[\begin{array}{c}
m \\
n
\end{array}\right]_{q}=\frac{(q ; q)_{m}}{(q ; q)_{n}(q ; q)_{m-n}}, \quad(t ; q)_{m}=\prod_{s=0}^{m-1}\left(1-t q^{s}\right),} \\
P_{n}^{(\alpha, \beta)}\left(\zeta ; q^{2}\right)=\sum_{r \geq 0} \frac{\left(q^{-2 n} ; q^{2}\right)_{r}\left(q^{2 \alpha+2 \beta+2 n+2} ; q^{2}\right)_{r}}{\left(q^{2} ; q^{2}\right)_{r}\left(q^{2 \alpha+2} ; q^{2}\right)_{r}}\left(q^{2} \zeta\right)^{r} .
\end{gathered}
$$

Corollary 4.9. For each $\ell \in(1 / 2) \mathbb{Z}_{+}$and $i, j \in I_{\ell}$, the following equality holds:

$$
w(\ell)_{i, j}^{*}=(-q)^{i-j} w(\ell)_{-i,-j} .
$$

Let $\sigma^{h}$ be the modular automorphism group for $h$. Then we have the following formula $[12,21,22$ :

$$
\sigma_{t}^{h}\left(w(\ell)_{r, s}\right)=q^{-2(r+s) i t} w(\ell)_{r, s} \quad \text { for all } t \in \mathbb{R} .
$$

4.5. Description of $L^{\infty}\left(\mathbb{T} \backslash S U_{q}(2)\right)$. Our aim is to prove the faithfulness of $\mathbb{T} \stackrel{\gamma^{\ell}}{\curvearrowright} Z\left(L^{\infty}\left(S U_{q}(2)\right)\right)$, which implies the factoriality of the standard Podles sphere $L^{\infty}\left(\mathbb{T} \backslash S U_{q}(2)\right)$ by Theorem 4.7. We let $Z:=Z\left(L^{\infty}\left(S U_{q}(2)\right)\right)$.

It is known that the spectrum of $\zeta=u^{*} u$ is quantized. More precisely, we obtain $\operatorname{Sp}(\zeta)=\left\{q^{2 k}\right\}_{k=0}^{\infty}\left[12,21\right.$. Hence there exist orthogonal projections $\left\{p_{k}\right\}_{k=0}^{\infty}$ in $C\left(S U_{q}(2)\right)$ such that

$$
\zeta=\sum_{k=0}^{\infty} q^{2 k} p_{k}
$$

LEMma 4.10. The set $\left\{p_{k}\right\}_{k=0}^{\infty}$ is a partition of unity in $L^{\infty}\left(S U_{q}(2)\right)$.

Proof. Let $p:=\sum_{k=0}^{\infty} p_{k}$ and $p^{\prime}:=1-p$. Using $\zeta x=q^{2} x \zeta$, we see that

$$
x p_{k}=p_{k+1} x \text {. }
$$

Summing up both sides over $k \geq 0$, we have $x p=\left(p-p_{0}\right) x$. Since $x x^{*}+\zeta=1$, we have $p_{0} x x^{*} p_{0}=0$, and $x p=p x$. Then

$$
\begin{aligned}
h\left(p^{\prime}\right) & =h\left(\left(x x^{*}+u u^{*}\right) p^{\prime}\right)=h\left(x x^{*} p^{\prime}\right) \\
& =h\left(x^{*} p^{\prime} \sigma_{-i}^{h}(x)\right)=q^{-2} h\left(x^{*} p^{\prime} x\right)=q^{-2} h\left(p^{\prime} x^{*} x\right) \\
& =q^{-2} h\left(p^{\prime}\left(x^{*} x+u^{*} u\right)\right)=q^{-2} h\left(p^{\prime}\right),
\end{aligned}
$$

and $h\left(p^{\prime}\right)=0$. Since $h$ is faithful, $p^{\prime}=0$. 
REMARK 4.11. We can compute $h\left(p_{k}\right)$ and directly verify the above result as follows. Recall the formula $h\left(\zeta^{n}\right)=\left(1-q^{2}\right) /\left(1-q^{2 n+2}\right)$ [12]. Since $\zeta^{n}$ converges to $p_{0}$ as $n \rightarrow \infty$ (in the norm topology), we have $h\left(p_{0}\right)=1-q^{2}$. Next using 4.4, we have

$$
h\left(x p_{k} x^{*}\right)=h\left(p_{k+1} x x^{*}\right)=h\left(p_{k+1}(1-\zeta)\right)=\left(1-q^{2 k+2}\right) h\left(p_{k+1}\right) .
$$

Thanks to $\sigma_{t}^{h}(x)=q^{2 i t} x$, we also obtain

$$
h\left(x p_{k} x^{*}\right)=h\left(p_{k} x^{*} \sigma_{-i}^{h}(x)\right)=q^{2} h\left(p_{k} x^{*} x\right)=q^{2} h\left(p_{k}\left(1-q^{2} \zeta\right)\right)=q^{2}\left(1-q^{2 k+2}\right) h\left(p_{k}\right) .
$$

Therefore, $h\left(p_{k+1}\right)=q^{2} h\left(p_{k}\right)$, and $h\left(p_{k}\right)=q^{2 k}\left(1-q^{2}\right)$. The summation of $\left\{h\left(p_{k}\right)\right\}_{k=0}^{\infty}$ is indeed equal to 1 .

THEOREM 4.12. The following hold:

1. $\gamma^{\ell}$ is a faithful action on $Z$;

2. $L^{\infty}\left(S U_{q}(2)\right)=Z \vee L^{\infty}\left(\mathbb{T} \backslash S U_{q}(2)\right)$;

3. $Z$ is $\mathbb{T}$-equivariantly isomorphic to $L^{\infty}(\mathbb{T})$;

4. $L^{\infty}\left(\mathbb{T} \backslash S U_{q}(2)\right)$ is the type $I_{\infty}$ factor.

Proof. Let $u=a|u|=a \zeta^{1 / 2}$ be the polar decomposition of $u$. By Lemma 4.10, $a a^{*}=$ $a^{*} a=1$. We show that $a \in Z$. Indeed, we have

$$
q x|u|^{2}=u x u^{*}=a|u| x|u| a^{*}=q a x|u|^{2} a^{*}=q a x a^{*}|u|^{2} .
$$

Again by Lemma 4.10 , we have $x=a x a^{*}$. The $*$-algebra $L^{\infty}\left(S U_{q}(2)\right)$ is generated by $x$ and $u$, so $a \in Z$. Since $\gamma_{z}^{\ell}(u)=z u$, we have $\gamma_{z}^{\ell}(a)=z a$ for all $z \in \mathbb{T}$. The other statements are trivial from Theorem 4.7

Hence Conjecture 4.5 holds for $S U_{q}(2)$. Although we have discussed and got the previous result using somewhat general results, we can directly obtain that from Woronowicz's classification of irreducible representations of the $C^{*}$-algebra $C\left(S U_{q}(2)\right)$ as follows.

Let us consider the tensor product von Neumann algebra $N:=L^{\infty}(\mathbb{T}) \otimes B\left(\ell_{2}\right)$. Define the four operators $x^{\prime}, v^{\prime}, u^{\prime}$ and $y^{\prime}$ in this algebra by

$$
\begin{aligned}
& x^{\prime}=z \otimes \sum_{k=0}^{\infty} \sqrt{1-q^{2 k+2}} e_{k+1, k}, \quad u^{\prime}=z \otimes \sum_{k=0}^{\infty}-q^{k} e_{k, k} \\
& v^{\prime}=\bar{z} \otimes \sum_{k=0}^{\infty} q^{k+1} e_{k, k}, \quad y^{\prime}=\bar{z} \otimes \sum_{k=0}^{\infty} \sqrt{1-q^{2 k+2}} e_{k, k+1},
\end{aligned}
$$

where $z \in L^{\infty}(\mathbb{T})$ is a canonical generating unitary. Then they satisfy the same relations as those of $C\left(S U_{q}(2)\right)$. Hence there exists a surjection $\pi: C\left(S U_{q}(2)\right) \rightarrow C^{*}\left(x^{\prime}, u^{\prime}, v^{\prime}, y^{\prime}\right)$ by universality.

Consider the tensor product state $h_{\mathbb{T}} \otimes \operatorname{Tr}_{\rho}$ on $N$, where $h_{\mathbb{T}}$ is the Haar state on $L^{\infty}(\mathbb{T})$ and the density is $\rho:=\sum_{k=0}^{\infty}\left(1-q^{2}\right) q^{2 k} e_{k k}$. We can verify $h=\left(h_{\mathbb{T}} \otimes \operatorname{Tr}_{\rho}\right) \circ \pi$ on the $*$-algebra generated by $x, u, v, y$ from Theorem 4.8 . Hence the surjection $\pi$ extends to the isomorphism between $L^{\infty}\left(S U_{q}(2)\right)$ and $C^{*}\left(x^{\prime}, u^{\prime}, v^{\prime}, y^{\prime}\right)^{\prime \prime}=N$. It is trivial that $\pi$ intertwines the torus actions $\gamma^{\ell}$ and $\beta \otimes \mathrm{id}$, where $\beta$ is the rotation on $L^{\infty}(\mathbb{Z})$. Hence $L^{\infty}\left(\mathbb{T} \backslash S U_{q}(2)\right)$ is isomorphic to $B\left(\ell_{2}\right)$. 
4.6. More complete description of $Z$. We close this article by giving generators of $Z$ for $S U_{q}(2)$ and studying their relations. Recall the torus actions $\gamma$ and $\gamma^{\ell}$ on $L^{\infty}\left(S U_{q}(2)\right)$,

$$
\left(\begin{array}{cc}
\gamma_{z}^{\ell}(x) & \gamma_{z}^{\ell}(u) \\
\gamma_{z}^{\ell}(v) & \gamma_{z}^{\ell}(y)
\end{array}\right)=\left(\begin{array}{cc}
z x & z u \\
\bar{z} v & \bar{z} y
\end{array}\right), \quad\left(\begin{array}{ll}
\gamma_{z}(x) & \gamma_{z}(u) \\
\gamma_{z}(v) & \gamma_{z}(y)
\end{array}\right)=\left(\begin{array}{cc}
z x & \bar{z} u \\
z v & \bar{z} y
\end{array}\right) \quad \text { for } z \in \mathbb{T}
$$

The following formulae are immediate from Theorem 4.8 .

$$
\gamma_{z}^{\ell}\left(w(\nu)_{i, j}\right)=z^{-2 i} w(\nu)_{i, j}, \quad \gamma_{z}\left(w(\nu)_{i, j}\right)=z^{-2 j} w(\nu)_{i, j}
$$

LEMMA 4.13. We have the following equalities:

1. $x w(\nu)_{r,-r}=\alpha_{\nu, r} w(\nu-1 / 2)_{r-1 / 2,-r-1 / 2}+\beta_{\nu, r} w(\nu+1 / 2)_{r-1 / 2,-r-1 / 2}$

2. $w(\nu)_{r,-r} x=\alpha_{\nu, r}^{\prime} w(\nu-1 / 2)_{r-1 / 2,-r-1 / 2}+\beta_{\nu, r}^{\prime} w(\nu+1 / 2)_{r-1 / 2,-r-1 / 2}$

3. $y w(\nu)_{r,-r}=\gamma_{\nu, r} w(\nu-1 / 2)_{r+1 / 2,-r+1 / 2}+\delta_{\nu, r} w(\nu+1 / 2)_{r+1 / 2,-r+1 / 2}$

4. $w(\nu)_{r,-r} y=\gamma_{\nu, r}^{\prime} w(\nu-1 / 2)_{r+1 / 2,-r+1 / 2}+\delta_{\nu, r}^{\prime} w(\nu+1 / 2)_{r+1 / 2,-r+1 / 2}$,

where the constants are given by

$$
\begin{aligned}
& \alpha_{\nu, r}=\gamma_{\nu, r}^{\prime}=q^{\nu+1} \frac{\sqrt{(\nu+r)_{q}(\nu-r)_{q}}}{(2 \nu+1)_{q}}, \quad \beta_{\nu, r}=\delta_{\nu, r}^{\prime}=q^{-\nu} \frac{\sqrt{(\nu+r+1)_{q}(\nu-r+1)_{q}}}{(2 \nu+1)_{q}}, \\
& \alpha_{\nu, r}^{\prime}=\gamma_{\nu, r}=q^{-\nu} \frac{\sqrt{(\nu+r)_{q}(\nu-r)_{q}}}{(2 \nu+1)_{q}}, \quad \beta_{\nu, r}^{\prime}=\delta_{\nu, r}=q^{\nu} \frac{\sqrt{(\nu+r+1)_{q}(\nu-r+1)_{q}}}{(2 \nu+1)_{q}},
\end{aligned}
$$

Proof. The element $x w(\nu)_{r,-r}$ is spanned by $w(\nu \pm 1 / 2)_{i, j}$. Using 4.5 , we see that there exist some complex numbers $\alpha$ and $\beta$ such that

$$
x w(\nu)_{r,-r}=\alpha w(\nu-1 / 2)_{r-1 / 2,-r-1 / 2}+\beta w(\nu+1 / 2)_{r-1 / 2,-r-1 / 2} .
$$

From Theorem 4.8 , we have

$$
\begin{gathered}
w(\nu)_{r,-r}=q^{-2 r(\nu-r)}\left[\begin{array}{c}
\nu+r \\
2 r
\end{array}\right]_{q^{2}} v^{2 r} P_{\nu-r}^{2 r, 0}\left(\zeta, q^{2}\right) \\
w(\nu-1 / 2)_{r-1 / 2,-r-1 / 2}=q^{-2 r(\nu-r-1)}\left[\begin{array}{c}
\nu+r-1 \\
2 r
\end{array}\right]_{q^{2}}^{1 / 2}\left[\begin{array}{c}
\nu+r \\
2 r
\end{array}\right]_{q^{2}}^{1 / 2} x v^{2 r} P_{\nu-r-1}^{2 r, 1}\left(\zeta, q^{2}\right) \\
w(\nu+1 / 2)_{r-1 / 2,-r-1 / 2}=q^{-2 r(\nu-r)}\left[\begin{array}{c}
\nu+r \\
2 r
\end{array}\right]_{q^{2}}^{1 / 2}\left[\begin{array}{c}
\nu+r+1 \\
2 r
\end{array}\right]_{q^{2}}^{1 / 2} x v^{2 r} P_{\nu-r}^{2 r, 1}\left(\zeta, q^{2}\right) .
\end{gathered}
$$

By comparing the constant term and the degree $(\nu-r)$ term of $\zeta$, we can obtain $\beta=q^{-\nu}(2 \nu+1)_{q}^{-1} \sqrt{(\nu+r+1)_{q}(\nu-r+1)_{q}}$ and $\alpha=q^{\nu+1}(2 \nu+1)_{q}^{-1} \sqrt{(\nu+r)_{q}(\nu-r)_{q}}$. Similarly we get the other equalities.

Let $r \in(1 / 2) \mathbb{Z}_{+}$and $Z_{-2 r}$ the spectral subspace for the action $\gamma^{\ell}$ for the eigenvalue $-2 r$. Since $Z \subset L^{\infty}\left(S U_{q}(2)\right)^{\sigma^{h}}$, an element $a \in Z_{-2 r}$ has the following expansion in $L^{2}\left(S U_{q}(2)\right)$ :

$$
a=\sum_{\nu=r}^{\infty} \lambda_{\nu, r} w(\nu)_{r,-r}
$$

where $\lambda_{\nu, r} \in \mathbb{C}$ and the summation is taken for $\nu=r, r+1, \ldots$ From the previous lemma, 
the commutativity of $a$ and $x$ implies

$$
\begin{aligned}
& \sum_{\nu \geq r} \lambda_{\nu, r}\left(\alpha_{\nu, r} w(\nu-1 / 2)_{r-1 / 2,-r-1 / 2}+\beta_{\nu, r} w(\nu+1 / 2)_{r-1 / 2,-r-1 / 2}\right) \\
& \quad=\sum_{\nu \geq r} \lambda_{\nu, r}\left(\alpha_{\nu, r}^{\prime} w(\nu-1 / 2)_{r-1 / 2,-r-1 / 2}+\beta_{\nu, r}^{\prime} w(\nu+1 / 2)_{r-1 / 2,-r-1 / 2}\right) .
\end{aligned}
$$

From this we get the recurrence formula $\lambda_{\nu+1, r}\left(\alpha_{\nu+1, r}-\alpha_{\nu+1, r}^{\prime}\right)=\lambda_{\nu, r}\left(\beta_{\nu, r}^{\prime}-\beta_{\nu, r}\right)$ whose solution is

$$
\lambda_{\nu, r}=\frac{(2 \nu+1)_{q}}{(\nu)_{q}(\nu+1)_{q}} \frac{(r)_{q}(r+1)_{q}}{(2 r+1)_{q}} \lambda_{r, r},
$$

where $(t)_{q}:=\left(q^{-t}-q^{t}\right) /\left(q^{-1}-q\right)$ for any $t \in \mathbb{R}$.

Lemma 4.14. For $r \in(1 / 2) \mathbb{Z}_{+}$, the following element $a_{r}$ is a well-defined unitary in $Z_{-2 r}$ and commutes with $x, u, v$ and $y$ :

$$
a_{r}=q^{-r}(r)_{q} \sum_{\nu=r}^{\infty} \frac{(2 \nu+1)_{q}}{(\nu)_{q}(\nu+1)_{q}} w(\nu)_{r,-r} .
$$

Proof. Since $\gamma^{\ell}$ is faithful on $Z$, the above elements have to be well-defined. Using the formulae $h\left(w(\nu)_{r,-r}^{*} w(\nu)_{r,-r}\right)=(2 \nu+1)^{-1} q^{2 r}$ and $(2 \nu+1)_{q}=(\nu+1)_{q}^{2}-(\nu)_{q}^{2}$, we have

$$
\begin{aligned}
h\left(a_{r}^{*} a_{r}\right) & =q^{-2 r}(r)_{q}^{2} \sum_{\nu=r}^{\infty} \frac{(2 \nu+1)_{q}^{2}}{(\nu)_{q}^{2}(\nu+1)_{q}^{2}} \cdot(2 \nu+1)_{q}^{-1} q^{2 r}=(r)_{q}^{2} \sum_{\nu=r}^{\infty} \frac{(2 \nu+1)_{q}}{(\nu)_{q}^{2}(\nu+1)_{q}^{2}} \\
& =(r)_{q}^{2} \sum_{\nu=r}^{\infty} \frac{(\nu+1)_{q}^{2}-(\nu)_{q}^{2}}{(\nu)_{q}^{2}(\nu+1)_{q}^{2}}=(r)_{q}^{2} \sum_{\nu=r}^{\infty}\left(\frac{1}{(\nu)_{q}^{2}}-\frac{1}{(\nu+1)_{q}^{2}}\right)=1 .
\end{aligned}
$$

Hence $a_{r}$ is a unitary.

Next we compare $a_{1 / 2}$ with $b$ that is a unitary of the polar decomposition of $v$, i.e. $v=b|v|=q b \zeta^{1 / 2}$. From this equality, we get $v^{*} b=q \zeta^{1 / 2}$. Using $h\left(p_{k}\right)=\left(1-q^{2}\right) q^{2 k}$, we obtain

$$
h\left(v^{*} b\right)=q h\left(z e^{1 / 2}\right)=q \sum_{k=0}^{\infty} q^{k}\left(1-q^{2}\right) q^{2 k}=\frac{q\left(1-q^{2}\right)}{1-q^{3}} .
$$

By definition, $a_{1 / 2}$ is equal to

$$
q^{-1 / 2}(1 / 2)_{q} \sum_{\nu=1 / 2}^{\infty} \frac{(2 \nu+1)_{q}}{(\nu)_{q}(\nu+1)_{q}} w(\nu)_{1 / 2,-1 / 2}=q^{-1 / 2}(1 / 2)_{q} \frac{(2)_{q}}{(1 / 2)_{q}(3 / 2)_{q}} v+\cdots .
$$

Using $h\left(v^{*} v\right)=q^{2} h(\zeta)=q^{2}\left(1+q^{2}\right)^{-1}$, we have

$$
h\left(v^{*} a_{1 / 2}\right)=q^{-1 / 2}(1 / 2)_{q} \cdot \frac{(2)_{q}}{(1 / 2)_{q}(3 / 2)_{q}} \cdot \frac{q^{2}}{1+q^{2}}=\frac{q\left(1-q^{2}\right)}{1-q^{3}} .
$$

Since $\operatorname{dim} Z_{-1}=1, a_{1 / 2}$ is a scalar multiple of $b$, but we have shown $a_{1 / 2}=b$ by the above calculation.

Therefore $v=a_{1 / 2}|v|$ is the polar decomposition. Next we compute $a_{1 / 2}^{k}, k \geq 1$, which has to be a scalar multiple of $a_{k / 2}$. In a similar way to the previous calculation, we obtain

$$
h\left(\left(v^{*}\right)^{k} a_{1 / 2}^{k}\right)=q^{k}\left(1-q^{2}\right)\left(1-q^{3}\right)^{-1}=h\left(\left(v^{*}\right)^{k} a_{k / 2}\right) .
$$

Summarizing our discussions, we have the following: 
THEOREM 4.15. Let $a_{r}$ be as above. Then the following hold:

1. $Z=\left\{a_{1 / 2}\right\}^{\prime \prime}$;

2. $v=a_{1 / 2}|v|$ is the polar decomposition;

3. $a_{1 / 2}^{k}=a_{k / 2}$ for all integer $k \geq 1$.

Acknowledgments. This research was supported by JSPS.

\section{References}

[1] H. Araki, D. Kastler, M. Takesaki and R. Haag, Extension of KMS states and chemical potential, Comm. Math. Phys. 53 (1977), 97-134.

[2] E. Bédos, R. Conti and L. Tuset, On amenability and co-amenability of algebraic quantum groups and their corepresentations, Canad. J. Math. 57 (2005), 17-60.

[3] E. Bédos, G. Murphy and L. Tuset, Co-amenability of compact quantum groups, J. Geom. Phys. 40 (2001), 130-153.

[4] E. Bédos, G. Murphy and L. Tuset, Amenability and coamenability of algebraic quantum groups, Int. J. Math. Math. Sci. 31 (2002), 577-601.

[5] S. Baaj and G. Skandalis, Unitaires multiplicatifs et dualité pour les produits croisés de $C^{*}$-algèbres, Ann. Sci. École Norm. Sup. (4) 26 (1993), 425-488.

[6] T. Hayashi, Harmonic function spaces of probability measures on fusion algebras, Publ. Res. Inst. Math. Sci. 36 (2000), 231-252.

[7] M. Izumi, Non-commutative Poisson boundaries and compact quantum group actions, Adv. Math. 169 (2002), 1-57.

[8] M. Izumi, S. Neshveyev and L. Tuset, Poisson boundary of the dual of $\mathrm{SU}_{q}(n)$, Comm. Math. Phys. 262 (2006), 505-531.

[9] G. I. Kac and V. G. Paljutkin, Finite ring groups, Trudy Moskov. Mat. Obšč. 15 (1966), $224-261$.

[10] L. I. Korogodski and Y. S. Soibelman, Algebras of Functions on Quantum Groups. Part I, Mathematical Surveys and Monographs 56, American Mathematical Society, Providence, RI, 1998.

[11] J. Kustermans and S. Vaes, Locally compact quantum groups in the von Neumann algebraic setting, Math. Scand. 92 (2003), 68-92.

[12] T. Masuda, K. Mimachi, Y. Nakagami, M. Noumi and K. Ueno, Representations of the quantum group $\mathrm{SU}_{q}(2)$ and the little q-Jacobi polynomials, J. Funct. Anal. 99 (1991), 357-386.

[13] P. Podleś, Quantum spheres, Lett. Math. Phys. 14 (1987), 193-202.

[14] P. Podleś, Symmetries of quantum spaces. Subgroups and quotient spaces of quantum $\mathrm{SU}(2)$ and $\mathrm{SO}(3)$ groups, Comm. Math. Phys. 170 (1995), 1-20.

[15] M. Takesaki, Conditional expectations in von Neumann algebras, J. Funct. Anal. 9 (1972), 306-321.

[16] R. Tomatsu, Amenable discrete quantum groups, J. Math. Soc. Japan 58 (2006), 949-964.

[17] R. Tomatsu, Compact quantum ergodic systems, J. Funct. Anal. 254 (2008), 1-83.

[18] R. Tomatsu, A characterization of right coideals of quotient type and its application to classification of Poisson boundaries, Comm. Math. Phys. 275 (2007), 271-296.

[19] R. Tomatsu and S. Vaes, work in progress.

[20] S. Vaes and N. Vander Vennet, to appear. 
[21] S. L. Woronowicz, Twisted SU(2) group. An example of a noncommutative differential calculus, Publ. Res. Inst. Math. Sci. 23 (1987), 117-181.

[22] S. L. Woronowicz, Compact quantum groups, in: Symétries quantiques (Les Houches, 1995), North-Holland, Amsterdam, 1998, 845-884. 